\title{
Modeling Simulation and Fabrication of Fiber Optic Pressure Sensor Pobe
}

\author{
M.L.Dongare \\ Department of Electronic Science \\ S.M.Joshi College, Hadapsar, \\ Pune - 4110 28, India
}

\author{
P.B.Buchade \\ Department of Electronic Science \\ Abasaheb Garware College \\ Pune - 411004, India
}

\author{
A.D.Shaligram \\ Department of Electronic Science, \\ University of Pune, \\ Pune - 411007, India
}

\begin{abstract}
Elastic element mechanical pressure gages consisting diaphragm are basically used for the differential pressure measurement. In FODPS, diaphragm acts as a reflector. A flexible disc of plastic is used as a diaphragm to convert the measuring pressure to the deflection of diaphragm. Concentric corrugations are designed to linearise the deflections according to pressure. Reference and measuring pressures are applied to control and experimental headspaces respectively. As the pressure changes in the experimental head space, the diaphragm is deform and displaces, and distance between fiber tip and plane of diaphragm changes and thus resultant reading is proportional to the differential pressure.
\end{abstract}

\section{Keywords}

Diaphragm, FODPS, pressure, sensor

\section{INTRODUCTION}

The FODPS is needed in physical, chemical, biochemical, analytical and biomedical applications [1-3]. The diaphragm deformation due to change in pressure was sensed by FODPS. The change in output due change in pressure was measured in terms of voltage. The application of fiber optic sensors in pressure measurement has the advantage that there is no physical contact with the process parameter to be measured.

Block diagram of FODPS is shown in figure.1. A flexible plastic disc is used as a diaphragm to convert the measuring pressure to the deflection of diaphragm. The FODPS is based on intensity modulation technique. Plastic fibers are used for its construction. The design aspect includes details of assembly of pressure chamber and sensing zone. The diaphragm deformation due to change in pressure was sensed by FODPS. The change in output voltage of the FODPS due to change in air pressure is acquired through ADC and directly displayed on LCD connected to a microcontroller.

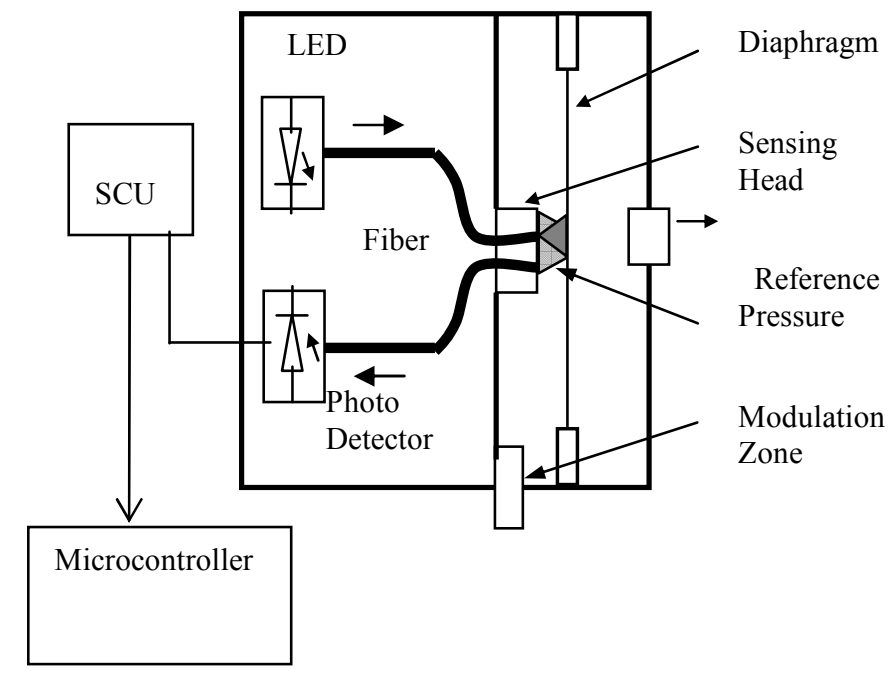

Figure1. Block diagram of FODPS

\section{Modeling and Simulation of FODPS}

Simulation is carried for varying a pressure at experimental side. The reference pressure was kept constant throughout the work. Initial pressure applied on both sides of the diaphragm was $21325 \mathrm{~Pa}$. Simulation values for pressure drop, on experimental sides varied from upper limit 21325 pa to lower limit rang 0 pa., because $21325 \mathrm{~Pa}$ is the partial pressure of pure distilled water. The geometrical information and corresponding changes through developed sensor are translated in to 3-D model. Diaphragm of plastic material of thickness $0.2 \mathrm{~mm}$, diameter 70 $\mathrm{mm}$ and applied pressure is from $1 \mathrm{kpa}$ to $21 \mathrm{kpa}$. For a small deflection range, the diaphragm deflections are mainly due to bending stresses in the plate. The deflection and the stresses developed due to uniformly acting pressure on diaphragm can be determined from the theory of plates.

We have used ANSYS as a FEA tool. It is a critical part in the whole structure of FODPS model of corrugated diaphragm. Estimation of its deflection was calculated using ANSYS. Finite element method is used for simulation of diaphragm under static pressure [4].

The overall equilibrium equation for linear structural static analysis [5] is given by 


$$
[\mathrm{S}](\mathrm{X})=\{\mathrm{P}\}
$$

Where $[\mathrm{S}]=$ Total stiffness matrix of diaphragm $=\mathrm{f}$ (geometry) $\mathrm{E}$, $\mathrm{E}=$ Young's Modulus $(\mathrm{X})=$ Nodal displacement vector $\quad\{\mathrm{P}\}=$ pressure vector Diaphragm is modeled as a shell element uniform thickness and pressure due to creation of vacuum remains the normal to element. Shell at center is used as a reflected region. Material properties like Young's modulus, Poisson's ratio and boundary condition like zero deformation is considered properly. First, nodes are considered along the positive $\mathrm{X}$-axis and these nodes are circularly patterned. Elements are created from nodes in the same fashion.

\section{Boundary Conditions}

Boundary conditions are, pressure drop at the reflecting surface (center of cup of diaphragm) and zero deformation at the supporting area of a probe. The nodes selected were from these boundaries. Finite element model of diaphragm with boundary condition and selected area is shown in figure 2(a)

Simulation with ANSYS program is used to define the shape of reflector when there is change in pressure. FE model of diaphragm with boundary condition is shown in figure 2 (b). It is divided into number of nodes. The diaphragm is divided in different regions - Reflector where the tip of fiber is mounted $\approx$ ( 0 t0 12 nodes), Corrugated area of diaphragm $\approx$ (13 to 80 nodes), Stiff or flat area $\approx(81$ to 95$)$, Supporting are to the wall of probe $\approx(96$ to 120$)$

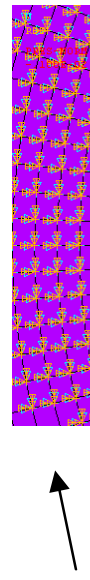

Support at

Probe wall

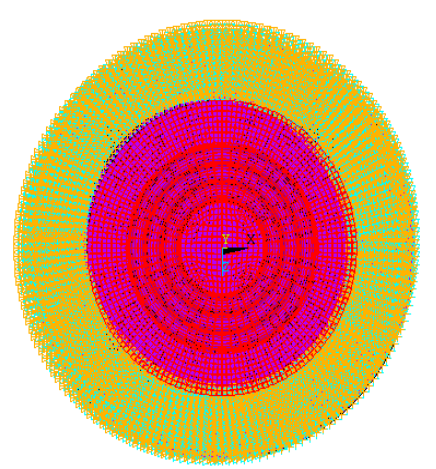

(b)
Figure 2 Finite element model of diaphragm with boundary condition

Pressure response on the diaphragm is shown in figure 3. Pressure is varied from $1 \mathrm{kpa}$ to $23 \mathrm{kpa}$ and corresponding displacement is recorded as shown in figure 4.

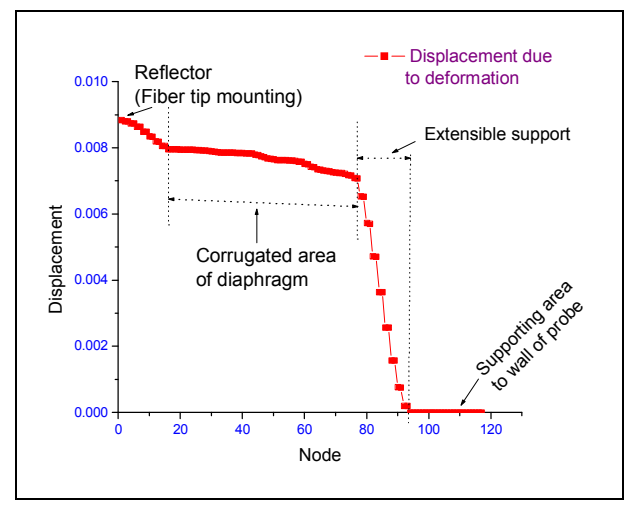

Figure.3: Pressure response on diaphragm nodes
Pressure drop detected area

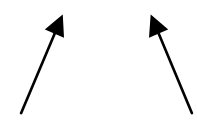

Corrugations

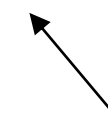

(a)

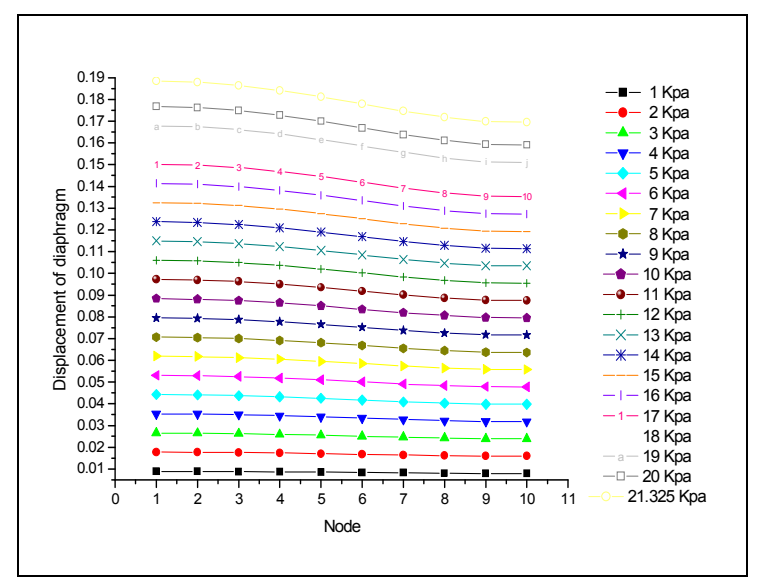

Figure.4 Displacement of diaphragm at various nodes with varying pressure 


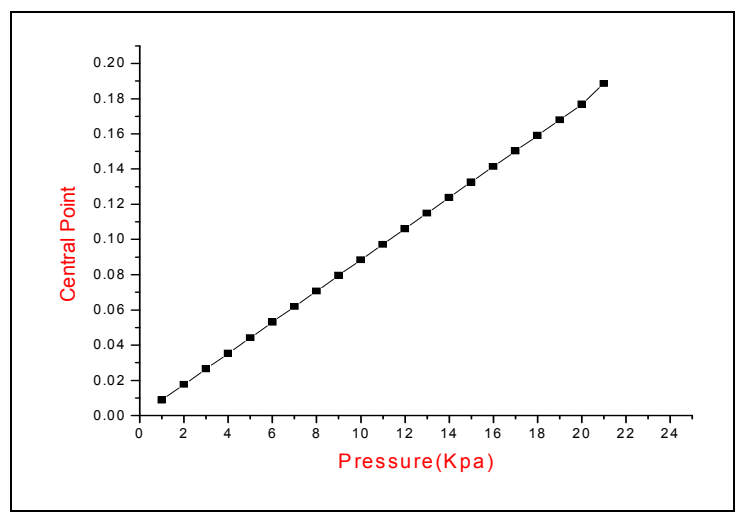

Figure.5 Center point displacement at various pressure

It founds that in the first region, there is a markable change in displacement. The center point displacement at various pressure is shown in figure 5. In the second region displacement is degraded. In the third stuff area region for the same pressure, it shows minimum displacement while in the last region i.e. at the supporting area of diaphragm to the wall of probe, it shows zero deformation.

FODPS was fabricated and used for the experimentation. Experiments were carried out for pressure range from $799.932 \mathrm{~Pa}$ to $3999.66 \mathrm{~Pa}$. It shows sensitivity of $0.000509 \mathrm{~V} / \mathrm{Pa}$ and linearity error -0.231 and precision of $79.818056 \%$. The simulation results are showing very good agreement with the experimental results.

Figure 6 shows the response of FODPS with diaphragm of plastic having thickness $0.2 \mathrm{~mm}$. Artificial pressure was applied

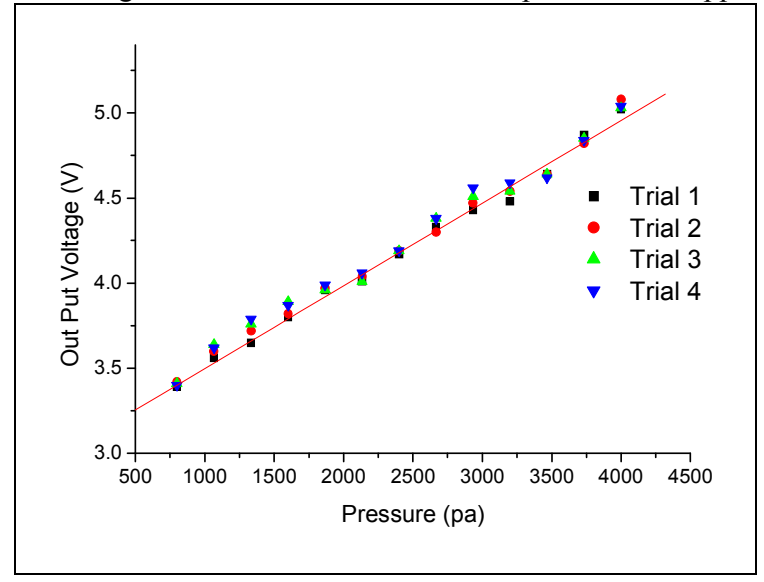

Figure.6 Response of pressure for various trials

on the diaphragm from one side and other side was kept open at atmospheric pressure. Initial output was adjusted to zero with the help of differential stage in the signal conditioning circuit. The output of FODPS increases with increase in pressure. The slope of the graph of output voltage at differential amplifier versus the applied pressure is the sensitivity of the FODPS.

\section{RESULT AND CONCLUSION}

It is hoped that this is new enhancement of FODPS which can be use effectively for the accurate pressure measurement comparatively than other different existing methods.

\section{Acknowledgements}

The authors express sincere thanks to Director of BCUD, University of Pune for providing a research grant, Principal, Rayat Shikshan Sanstha's S.M Joshi college, Hadapsar Pune-28, for permitting us to carry out the research work and providing the necessary facilities.

\section{REFERENCES:}

[1] Yuan Libo, Ruan Shunling and Pan Jian 1993. 'Automatic compensation fiber optic differential pressure sensor', Sensors and Actuators: A, 36 pp. 183-185

[2] O B Wright, D Largeau, 1987. "Fibre-optic differential pressure sensor", J. Phys. E: Sci. Instrum. 20 pp. 46-51

[3] Berthold J.W. Ghering W.L. and Varshneya, 1987 "Design and characterization of a high temperature fiber optic pressure transducer", J. light wave technology, 5 pp. 870 876

[4] Arrendale H., 1973 "Precession Pressure Measurement", Instruments and Control Systems, volume 13,10

[5] Shelke, Kiran Tamadaddi, P.B. Buchade and A.D. Shaligram, 2005 " Finite Element Modeling and Simulation of Fiber optical based load cell (FOLC) sensor", Proc. SPIE Vol.5852 pp. 384-389. 